
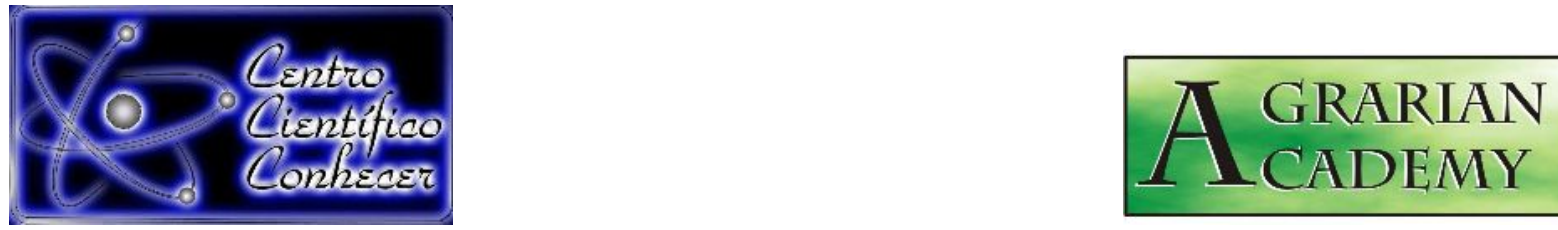

\title{
ESTUDO DA POROSIDADE E DUREZA DA MADEIRA DE SERINGUEIRA (Hevea brasiliensis Muell. Arg.)
}

Douglas Lamounier Faria'; Isabelle Cristine de Carvalho Terra' ${ }^{1}$; Kleber Magalhães

Oliveira $^{2}$; Ana Carolina Corrêa Furtini ${ }^{3}$; José Benedito Guimarães Júnior ${ }^{4}$

1. Pós-Graduando em Engenharia de Biomateriais - Depto de Ciências Florestais -

Universidade Federal de Lavras - Lavras/MG - Brasil

2. Graduando em Engenharia Florestal - Depto de Ciências Florestais - Universidade Federal de Lavras - Lavras/MG - Brasil

3. Graduada em Engenharia Civil - Centro Universitário de Lavras - Lavras/MG - Brasil

4. Prof. Dr. em Ciência e Tecnologia da Madeira - Depto de Engenharia - Universidade

Federal de Lavras - Lavras/MG - Brasil

E-mail para correspondência: douglas.lamounier@yahoo.com

Recebido em: 14/07/2018 - Aprovado em: 28/07/2018 - Publicado em: 31/07/2018

DOI: 10.18677/Agrarian_Academy_2018a36

O presente trabalho propõe avaliar a porosidade e a dureza da madeira de Hevea brasiliensis tratada com dicromato de cobre cromatado (CCB) e Pentaclorofenol (Pentox), visando a utilização da madeira em situações estruturais. Para realização dos ensaios foram cortadas três árvores do clone de seringueira $\mathrm{Pb} 235$, com 25 anos de idade e DAP superior a $50 \mathrm{~cm}$. Foram avaliadas as propriedades fisicas de umidade, densidade aparente e porosidade, e a propriedade mecânica módulo de elasticidade (MOE) nas direções paralela e perpendicular, através do ensaio de Dureza Janka. Em relação aos resultados obtidos para MOE, os tratamentos apresentaram valores médios estatisticamente iguais. Porém, ao comparar os tratamentos, foi observado que as madeiras apresentaram maiores valores médios para a direção paralela. No entanto, em relação aos resultados de porosidade para os diferentes tratamentos realizados na madeira de seringueira, houve diferença estatística entre eles. A madeira sem tratamento preservativo apresentou os maiores valores médios de porosidade $(52,45 \%)$, seguido pelo tratamento com Pentox $(48,81 \%)$ e posteriormente pela madeira tratada com CCB $(46,45 \%)$. Os resultados obtidos permitem colocar a madeira de seringueira como uma espécie promissora na utilização em situações estruturais, como o uso em dormentes de vias férreas.

PALAVRAS-CHAVE: Dureza Janka; módulo de elasticidade e tratamentos químicos.

\section{STUDY OF THE POROSITY AND HARDNESS OF THE WOOD OF RUBBER WOOD (Hevea brasiliensis Muell. Arg.)}

ABSTRACT

The present work proposes to evaluate the porosity and hardness of the wood of Hevea brasiliensis treated with chrome copper dichromate (CCB) and AGRARIAN ACADEMY, Centro Científico Conhecer - Goiânia, v.5, n.9; p. 3682018 
Pentachlorophenol (Pentox), aiming at the use of wood in structural situations. For the accomplishment of the tests three trees of the rubber wood clone $\mathrm{Pb} 235$, with 25 years of age and $\mathrm{DBH}$ greater than $50 \mathrm{~cm}$ were cut. The physical properties of moisture, apparent density and porosity, and the mechanical property modulus of elasticity (MOE) in the parallel and perpendicular directions were evaluated through the Janka hardness test. Regarding the results obtained for MOE, the treatments presented statistically equal mean values. However, when comparing the treatments, it was observed that the wood presented higher average values for the parallel direction. However, in relation to the porosity results for the different treatments performed on the rubber tree, there was statistical difference between them. The wood without preservative treatment had the highest average values of porosity $(52.45 \%)$, followed by treatment with Pentox (48.81\%) and later by wood treated with CCB $(46.45 \%)$. The results obtained allow to place the rubber wood as a promising species in the use in structural situations, such as the use in railway pieces.

KEYWORDS: Chemical treatments; modulus of elasticity; Janka hardness.

\section{INTRODUÇÃO}

A Hevea brasiliensis (seringueira) é uma espécie nativa da Amazônia e apresenta elevada importância econômica no Brasil, por ser amplamente utilizada na obtenção de látex para produção de borracha (IWAKIRI et al., 2017). No Brasil, após o ciclo de extração do látex, plantações de seringueiras são reformuladas e a madeira dessas plantações são tradicionalmente usadas como fonte de energia barata (EUFRADE JUNIOR et al., 2015).

Por ser um material heterogêneo, a madeira apresenta propriedades físicas, químicas, anatômicas e mecânicas que podem variar entre espécies, dentro da mesma espécie e até na mesma árvore. Por isso, conhecer suas propriedades e a interação dessas propriedades é fundamental para a correta utilização dessa matéria-prima (PALERMO et al., 2013).

O conhecimento das propriedades físicas e mecânicas das madeiras é fundamental para definir adequadamente as aplicações a que serão destinadas e dimensionar, com segurança, as partes componentes de uma estrutura com esse material. Para se utilizar a madeira como elemento estrutural, devem ser tomadas precauções visando a segurança bem como a garantia que o material irá resistir às solicitações que irá vir sofrer durante sua utilização (ALMEIDA et al., 2013).

O conhecimento das relações existentes entre a estrutura anatômica da madeira com suas propriedades físicas ou mecânicas é importante para seu uso adequado (MOTTA et al., 2014). A porosidade define a porcentagem de vazio dentro do volume aparente (COSTA, 2006). A impregnação de adesivo está associada à porosidade da madeira que é maior em madeiras de baixa densidade do que as de alta densidade, o que facilita a penetração do adesivo (MUZEL, 2013). Sendo assim, a porosidade favorece o espalhamento ou absorção do líquido na madeira.

A dureza de um material pode ser definida como a resistência que um corpo sólido apresenta à penetração de outro corpo sólido através da aplicação de uma determinada força, ou então como a capacidade de um corpo de resistir à deformação (CALONEGO et al., 2017).

Segundo a norma Projeto de Estruturas de Madeira; NBR 7190 (ABNT, 1997), a dureza Janka é um método para caracterizar a dureza, no qual as forças devem ser aplicadas nas direções paralela e perpendicular às fibras. Sendo estabelecida pela força necessária para penetrar, até a metade do diâmetro, de uma esfera de aço de diâmetro $11,28 \mathrm{~mm}$. O carregamento deve ser monotônico crescente 
aplicado até que a esfera penetre a uma profundidade igual ao seu raio, em um período de pelo menos um minuto.

Conforme Pogetto et al. (2006) a importância da determinação da dureza da madeira, nas direções paralela e perpendicular às fibras, com vistas ao emprego em dormentes. Assinalam que a dureza Janka vem ganhando destaque no conjunto das informações essenciais usadas para avaliar a qualidade da madeira e seu potencial tecnológico. Apontam que a relação entre a dureza paralela e a dureza normal às fibras (determinadas pelo método Janka) se aproxima de 1, com alguma tendência de aumento para as densidades mais elevadas.

A madeira utilizada em construções e marcenaria, quando não tratada, fica sujeita ao ataque de agentes biológicos xilófagos, a exemplo dos fungos e térmitas (cupins), os quais provocam apodrecimento, manchas e destruição das peças. A utilização de técnicas preservativas protege a madeira do ataque desses agentes, prolongando a vida útil. Um desses produtos é o borato de cobre cromatado (CCB), também conhecido como Wolmanit $\mathrm{CB}$, é um produto que surgiu na Alemanha, como tentativa de substituir o arsênio das formulações de arseniato de cobre cromatado (CCA) (SILVA, 2007).

Os boratos possuem propriedades fungicidas, inseticidas e ignífugas e têm sido caracterizados como preservativos muito eficientes quando usados em madeiras que não fiquem em contato com o solo. É um produto que pode ser usado em autoclave ou em métodos caseiros, sob difusão. Porém o CCB tem uma sensível perda quanto à resistência à lixiviação, provocando queda na eficiência de proteção contra os insetos, sobretudo para madeira que ficará exposta por extenso período (SILVA, 2007).

Outro método preservativo e mais simples é realizando o pincelamento da madeira. Um dos principais produtos utilizados é o pentaclorofenol, um produto organoclorado, obtido pela reação entre o fenol e o cloro $\left(\mathrm{C}_{6} \mathrm{Cl}_{5} \mathrm{OH}\right)$ até a completa substituição de todos os átomos de hidrogênio por átomos de cloro. É um produto insolúvel em água, mas solúvel em solventes orgânicos, apresentando excelente ação fungicida e uma boa ação inseticida. A importância como preservativo de madeira se deve, principalmente, à alta toxidez aos agentes xilófagos e grande resistência à lixiviação, por ser praticamente insolúvel em água à temperatura ambiente (SILVA, 2007).

Desta forma, o objetivo do presente trabalho foi avaliar a porosidade e o módulo de elasticidade (MOE) no ensaio de dureza Janka no sentido paralelo e perpendicular da madeira de Hevea brasiliensis tratada com CCB e Pentox, visando a utilização da madeira em situações estruturais, como é o caso de dormentes para via férrea.

\section{Obtenção e preparo da madeira de seringueira}

\section{MATERIAL E MÉTODOS}

O experimento foi instalado em Lavras, no Sul do Estado de Minas Gerais, a $900 \mathrm{~m}$ de altitude, latitude de 2114'S e longitude de 450' W. Apresenta clima do tipo CWB segundo classificação de Köppen. A temperatura média anual é de 19,4 $\mathrm{C}$, com média da máxima de $26,1^{\circ} \mathrm{C}$ e média da mínima de $14,8^{\circ} \mathrm{C}$.

Foram cortadas três árvores do clone de seringueira $\mathrm{Pb} 235$, (Hevea brasiliensis - Muell. Arg) com 25 anos de idade e DAP (Diâmetro a altura de 1,30m do solo) superior a $50 \mathrm{~cm}$ no campus da Universidade Federal de Lavras (UFLA), localizada em Lavras, no Sul do Estado de Minas Gerais. De cada árvore, foram 
retirados toretes de $1,30 \mathrm{~m}$ a partir da base, em que parte foi tratada com CCB em até 24 horas após o corte das árvores.

Para o tratamento dos toretes, foi utilizado um produto hidrossolúvel, fungicida e inseticida à base de borato de cobre cromatado (CCB-Óxido), apresentando 2,5\% de ingredientes ativos, possuindo boa mobilidade na madeira, sendo indicado para o tratamento de material verde. Empregou-se o método de substituição da seiva, por transpiração radial (Figura 1). Optou-se por essa técnica pela simplicidade de manuseio. Após 20 dias para fixação do preservativo CCB, os toretes foram seccionados em pranchões centrais. Já para o tratamento com inseticida à base de pentaclorofenol, foi utilizado o produto Pentox (fabricado pela Montana Química S/A, São Paulo, Brasil), a aplicação do produto foi realizada por pincelamento na madeira, após secção e confecção dos corpos de prova para os ensaios.

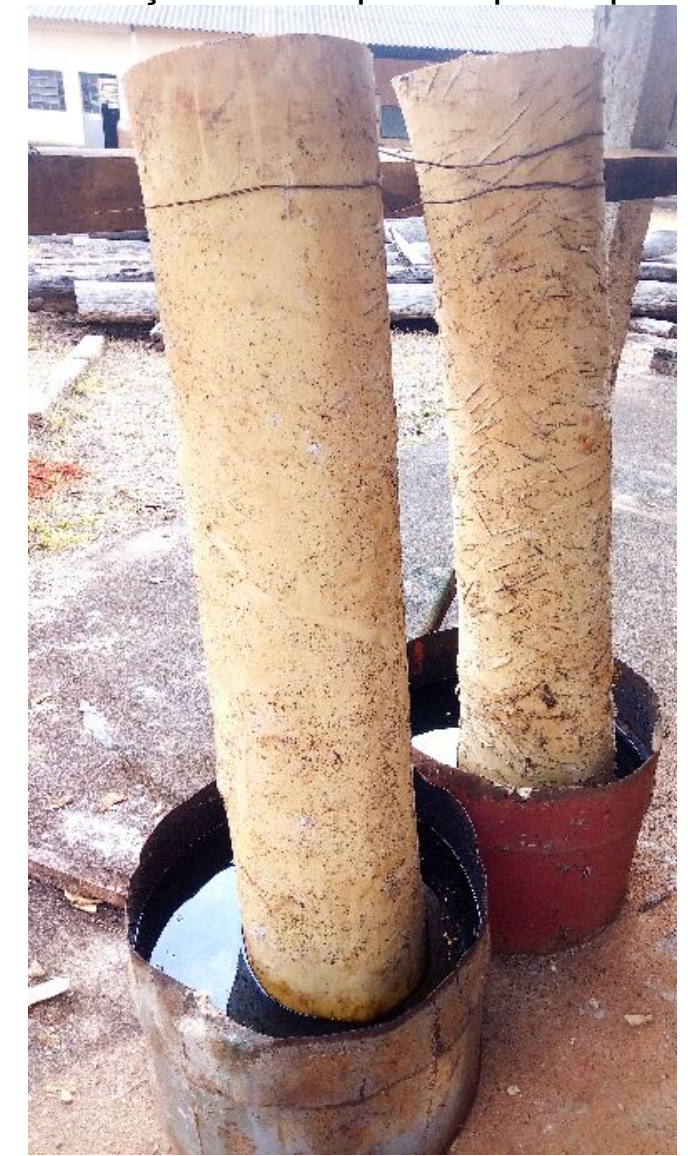

FIGURA 1. Tratamento dos toretes com CCB pelo método de substituição de seiva.

Os pranchões centrais foram então acondicionadas em ambiente com temperatura de $22 \pm 2{ }^{\circ} \mathrm{C}$ e umidade relativa de $65 \pm 5 \%$. Posteriormente, os corpos de prova foram obtidos com as dimensões de acordo com a Tabela 1 abaixo.

TABELA 1 - Detalhamento experimental para os ensaios realizados na madeira de seringueira.

\begin{tabular}{lll}
\hline Propriedade & Dimensões $(\mathrm{cm})$ & Norma \\
\hline Umidade & $2,0 \times 3,0 \times 5,0$ & NBR $7190(1997)$ \\
Densidade aparente & $5,0 \times 5,0 \times 15,0$ & \\
Dureza Janka & & \\
\hline
\end{tabular}

AGRARIAN ACADEMY, Centro Científico Conhecer - Goiânia, v.5, n.9; p. 3712018 
A umidade e densidade da madeira de seringueira foi obtida de um total de 15 corpos de prova para cada ensaio, em cada um dos três tratamentos. Para a determinação do módulo de elasticidade (MOE) nas direções paralela e perpendicular no ensaio de Dureza Janka, foram utilizados 20 corpos de prova por tratamento.

O ensaio de Dureza Janka foi realizado em uma prensa servo-elétrica da marca Arotec (Figura 2), dotada de uma célula de carga de $300 \mathrm{kN}$, localizada no Departamento de Ciências Florestais, na Universidade Federal de Lavras (UFLA).

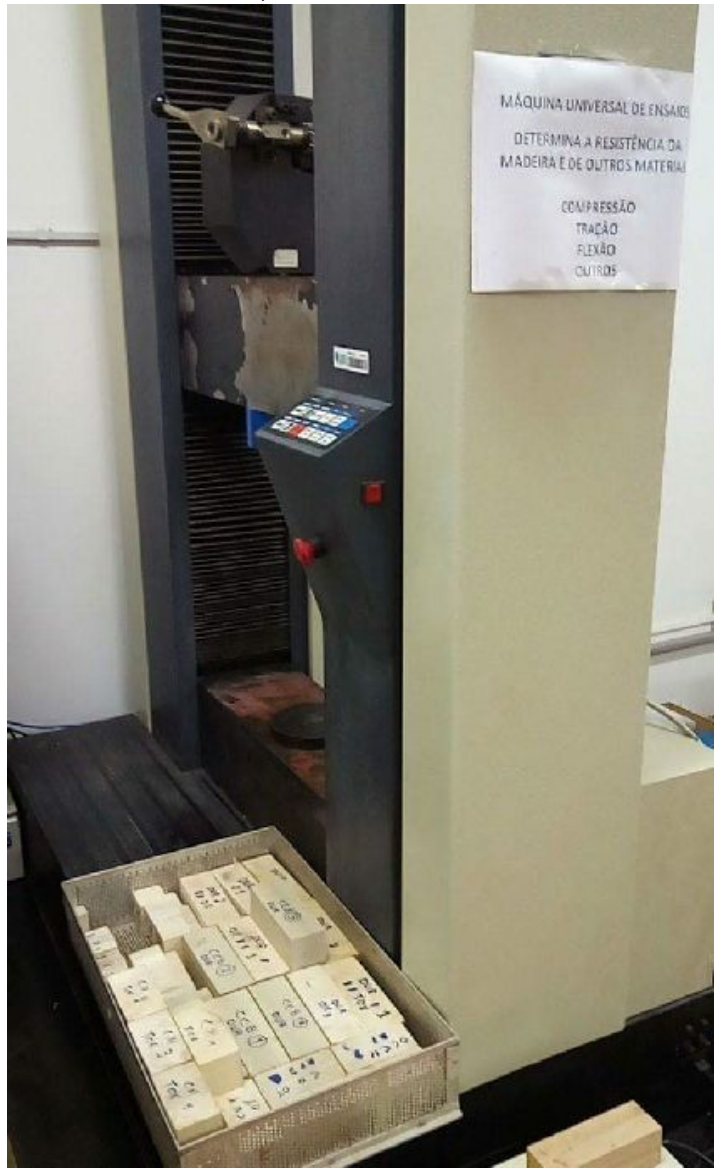

FIGURA 2. Prensa utilizada para realização dos ensaios de dureza Janka.

Para a determinação da porosidade da madeira foi calculada por meio da fórmula proposta por MacLean (1952), em que a porosidade da madeira foi determinada para cada um dos tratamentos, a qual estabelece que a porosidade está em função da densidade e do teor de umidade da madeira, conforme equação 1.

$$
P m=1-\text { DENSfv }\left(0,667+0,01{ }^{*} T U f v\right){ }^{*} 100
$$

Em que:

Pm: porosidade da madeira (\%);

DENSfv: densidade aparente da madeira $\left(\mathrm{g} / \mathrm{cm}^{3}\right)$;

TUfv: teor de umidade da madeira.

As propriedades foram avaliadas em um delineamento inteiramente casualizado. Para a avaliação da umidade, densidade aparente, porosidade e 
dureza Janka, foi realizada a análise de variância e teste de média Tukey, ambos a 5\% de significância, utilizando o software Sisvar.

\section{RESULTADOS E DISCUSSÃO}

$\mathrm{Na}$ Tabela 2 podem ser observados os valores médios de densidade aparente e umidade da madeira de Hevea brasiliensis para os três tratamentos avaliados.

TABELA 2 - Densidade aparente e umidade dos diferentes tipos de tratamentos.

\begin{tabular}{ccc}
\hline \multirow{2}{*}{ Tratamento } & $\begin{array}{c}\text { Densidade } \\
\text { Aparente }\end{array}$ & Umidade \\
\cline { 2 - 3 } & $\mathbf{( \% )}^{(\%)}$ & \\
\hline Sem tratamento & $0,624^{(0,014)} \mathrm{A}$ & $11,298^{(0,537)} \mathrm{B}$ \\
CCB & $0,654^{(0,016)} \mathrm{A}$ & $12,272^{(0,434)} \mathrm{B}$ \\
Pentox & $0,637^{(0,022)} \mathrm{A}$ & $10,578^{(0,131)} \mathrm{A}$ \\
\hline
\end{tabular}

*Médias seguidas de mesma letra, na coluna, não diferem estatisticamente entre si, pelo teste Tukey a $5 \%$ de significância. Desvio padrão entre parênteses.

Conforme observado na Tabela 2, todos os tratamentos apresentaram valores médios estatisticamente iguais para densidade aparente, sendo estes de 0,624 a $0,654 \mathrm{~g} / \mathrm{cm}^{3}$. Para em razão de sua massa e do volume que inclui o material lenhoso. Braz et al. (2013) Moreschi (2005) as densidades aparente e básica é utilizado quando se calcula a massa específica da madeira frisam que é uma das propriedades mais importantes da madeira, pois quanto maior a densidade da madeira, maiores serão suas propriedades mecânicas.

A densidade fornece indicações gerais sobre a utilidade, transporte e manuseio das espécies madeireiras. Trianoski et al. (2013) relatam que a massa específica média da madeira de seringueira é maior que a madeira de Pinus, em torno de $0,450 \mathrm{~g} / \mathrm{cm}^{3}$.

Raia (2017), avaliou-se painéis de Hevea brasiliensis com tratamentos sem extração e com extração de látex, apresentou-se densidade média aparente respectivamente, $0,674 \mathrm{~g} / \mathrm{cm}^{3}$ e $0,655 \mathrm{~g} / \mathrm{cm}^{3}$. Resultados semelhantes foram obtidos por Iwakiri et al., (2017) e Riyaphan et al., (2015) encontrando valores médios para densidade aparente de $0,572 \mathrm{~g} / \mathrm{cm}^{3}$ e $0,59 \mathrm{~g} / \mathrm{cm}^{3}$, para o clone RRIM 600, respectivamente. Isso mostra que os resultados encontrados nesse trabalho estão próximos aos encontrados na literatura.

Em relação à umidade para os diferentes tratamentos realizados na madeira de seringueira, observa-se na Tabela 2 que houve diferença estatística entre os tratamentos, as madeiras sem tratamento preservativo e tratada com CCB apresentaram os maiores valores médios. A umidade final ficou próxima de $12 \%$, valor esse referente à umidade de equilíbrio higroscópico da região.

Com os valores de umidade e densidade aparente foi calculada a porosidade da madeira para os tratamentos. Os resultados para a propriedade estão apresentados na Tabela 3 a seguir: $\mathrm{Na}$ Tabela 3, pode-se observar os valores médios de porosidade para os diferentes tratamentos da madeira de seringueira. 
TABELA 3 - Valores médios de porosidade para os três tratamentos da madeira.

\begin{tabular}{ll}
\hline Tratamento & Porosidade $(\%)$ \\
\hline Pentox & $50,62^{(0,16)} \mathrm{B}$ \\
Sem tratamento & $51,54{ }^{(0,14)} \mathrm{C}$ \\
CCB & $48,39^{(0,10)} \mathrm{A}$ \\
\hline
\end{tabular}

*Médias seguidas de mesma letra, na coluna, não diferem estatisticamente entre si, pelo teste Tukey a $5 \%$ de significância. Desvio padrão entre parênteses.

Conforme observado na Tabela 3, os três tratamentos da madeira diferiram estatisticamente entre si. A madeira sem tratamento preservativo apresentou os maiores valores médios de porosidade $(52,45 \%)$, seguido pelo tratamento com Pentox $(48,81 \%)$ e posteriormente pela madeira tratada com CCB $(46,45 \%)$. De acordo com Iwakiri (2005), a frequência de vasos está relacionada com a porosidade, que se relaciona com a densidade, o que influencia a penetração dos adesivos na madeira, bem como as características mecânicas da madeira. A dureza da madeira de seringueira foi determinada pelo método proposto por Janka, sendo os resultados apresentados na Tabela 4 abaixo:

TABELA 4 - Módulo de elasticidade (MOE) para o ensaio de dureza Janka dos diferentes tipos de tratamentos.

\begin{tabular}{|c|c|c|}
\hline \multirow{2}{*}{ Tratamento } & Paralelo & Perpendicular \\
\hline & \multicolumn{2}{|c|}{ MOE (MPa) } \\
\hline Pentox & $116,96^{(12,03)} \mathrm{Ab}$ & $74,67^{(7,77)} \mathrm{Aa}$ \\
\hline Sem tratamento & $127,98^{(15,44)} \mathrm{Ab}$ & $73,67^{(7,57)} \mathrm{Aa}$ \\
\hline CCB & $136,19^{(25,95)} \mathrm{Ab}$ & $66,71^{(7,58)} \mathrm{Aa}$ \\
\hline
\end{tabular}

*Médias seguidas de mesma letra, na coluna, não diferem estatisticamente entre si, pelo teste Tukey a $5 \%$ de significância. Desvio padrão entre parênteses.

Observando os resultados apresentados na Tabela 3 acima, é verificado que não houve diferença significativa ao nível de $5 \%$ para o módulo de elasticidade (MOE) para as direções paralelo e perpendicular entre os tratamentos. Porém, ao comparar os tratamentos, foi observado que as madeiras apresentaram maiores valores médios para a direção paralela no ensaio de dureza Janka.

O comportamento observado para os diferentes tratamentos nas duas variáveis analisadas (direções paralelo e perpendicular), reitera a relação que ocorre entre a densidade da madeira e a dureza Janka. Foi observado na Tabela 2 que não houve diferença significativa para densidade aparente para os tratamentos avaliados, o mesmo se mostrou para a dureza Janka.

Os resultados encontrados nesse trabalho foram próximos aos obtidos por Riyaphan et al., (2015) estudando a variabilidade química e mecânica de árvores de seringueira, onde os autores obtiveram valores médios de $60 \mathrm{MPa}$ para a dureza no sentido perpendicular de árvores de seringueira do clone RRIM 600. 


\section{CONCLUSÃO}

Foram observados maiores valores para a porosidade sem tratamento preservativo realizado. Esse fato pode ser explicado pela madeira não apresentar entupimentos dos poros pelas soluções preservativas, porém há a necessidade de uma análise específica para confirmar tal afirmação, por meio de obtenção de cortes anatômicos da madeira. Como a porosidade está diretamente relacionada com as propriedades mecânicas, quanto maior a porosidade maior será os índices de vazios e consequentemente menor será a resistência mecânica.

Os valores obtidos para a dureza permitem colocar a madeira de seringueira como uma espécie promissora na utilização em situações estruturais, como é o caso proposto nesse trabalho, dos dormentes de vias férreas. Foi observado que independente do tratamento preservativo realizado, não houve diferença estatística entre os tratamentos. Tal resultado é de extrema importância, uma vez que a madeira de seringueira é muito suscetível ao ataque de agentes xilófagos, e como a finalidade é a utilização da madeira em dormentes, há a necessidade de tratamento da madeira para que a mesma possua uma durabilidade maior. Porém, há a necessidade de conhecer a fundo as propriedades da madeira, como a resistência à compressão paralela às fibras e a anatomia da mesma, afim de se estabelecer bons estimadores das propriedades indispensáveis para definir seu sucesso (ou não) para aplicação na fabricação de dormentes.

\section{REFERÊNCIAS}

ALMEIDA, D. H; SCALIANTE, R. M.; MACEDO, L. B.; MACEDO, A. N. ; DIAS, A. A. ; CHRISTOFORO, A. L. ; CALIL JUNIOR, C. Caracterização completa da madeira da espécie amazônica Paricá (Schizolobium amazonicum HERB) em peças de dimensões estruturais. Revista Árvore (Impresso), v. 37, p. 1175-1181, 2013.

ABNT - Associação Brasileira de Normas técnicas . Projeto de estruturas de madeira. Rio de Janeiro, 1997. (NBR 7190).

BRAZ, R. L.; OLIVEIRA, J. T. S.; RODRIGUES, B. P.; ARANTES, M. D. C. Propriedades físicas e mecânicas da madeira de Toona ciliata em diferentes idades. Floresta, Curitiba, PR, v. 43, n. 4, p. 663 - 670, out. / dez. 2013.

CALONEGo, F. W.; SEVERO, E. T. D.; BRITO, A. F.; RODRIGUES, P. L. M.; PELOZZI, M. M. A. Correlação entre dureza janka e outras propriedades da madeira de Eucalyptus grandis modificada termicamente. III Congresso Latinoamericano de Estructuras de Madeira + III Congreso Ibero-Latinoamericano de la Madera en la Construcción, Buenos Aires, 2017.

COSTA, V. C. Caracterização físico-energética da madeira e produtividade de reflorestamentos de clones de híbridos de Eucalyptus grandis x E. urophylla. Tese apresentada à Faculdade de Ciências Agronômicas da UNESP-Botucatu: 2006. Disponível em: <http://www.pg.fca.unesp.br/Teses/PDFs/Arq0043.pdf>.

EUFRADE JUNIOR, H. J.; OHTO, J. M.; SILVA, L. L.; PALMA, H. A. L.; BALLARIN, A. W. Potential of rubberwood (Hevea brasiliensis) for structural use after the period of latex extraction: a case study in Brazil. Journal Wood Science, v. 61, p. 384-390, 2015. DOI 10.1007/s10086-015-1478-7

IWAKIRI, S. Painéis de madeira reconstituída. Curitiba: Fupef, 2005. 247 p. 
IWAKIRI, S.; TRIANOSKI, R.; WEBER, A. M.; BONFATII JUNIOR, E. A.; PEREIRA, G. F.; BUENO, J. A.; CECHIN. L.; RAIA, R. Z. Efeitos do tratamento de partículas e aceleradores de endurecimento na produção de painéis cimento-madeira de Hevea brasiliensis. Floresta, Curitiba, PR, v. 47, n. 3, p. 289-296, jul./set. 2017. DOI: 10.5380/rf.v47i1.51636

MACLEAN, J. D. Preservative treatment of wood by pressure metrods. Madison: U. S. Department of Agriculture, 1952. 160 p.

MORESCHI, J. C. Propriedades tecnológicas da madeira, 2005. http://www.madeira.ufpr.br/publicacoes/propriedadesdamadeira2010.pdf

MOTTA, J. P.; OliVEIRA, J. T. S.; BRAZ, R. L.; DUARTE, A. P. C.; ALVES, R. C. Caracterização da madeira de quatro espécies florestais. Ciência Rural, Santa Maria, v.44, n.12, p.2186-2192, dez, 2014. http://dx.doi.org/10.1590/01038478 cr20130479

MUZEL, S.D. Madeira deHevea brasiliensiscomo matéria prima para a produção de chapa de partículas aglomeradas.Trabalho de conclusão de curso (bacharelado - Engenharia Industrial Madeireira) - Universidade Estadual Paulista, Campus de Itapeva, $2013 . \quad$ Disponível em: <https://repositorio.unesp.br/bitstream/handle/11449/142901/000867491.pdf?se quence $=1>$.

PALERMO, G. P. M.; LATORRACA, J. V. F.; SEVERO, E. T. D.; NASCIMENTO, A. M.; REZENDE, M. A. Delimitação entre os lenhos juvenil e adulto de Pinus elliottii Engelm. Revista Árvore, Viçosa-MG, v. 37, n. 1, p. 191-200, 2013.

POGETTO, M.H.F.A.; BALLARIN, A.W.; COLENCI, R.A. Relações entre a dureza paralela e normal às fibras em madeiras. In: Encontro Brasileiro em madeira e em estruturas de madeira, 10, 2006, Águas de São Pedro. Anais... Botucatu:UNESP, 2006. (CDROM).

RAIA, R. Z. Laminação e produção de painéis compensados de Hevea brasiliensis - Clone RRIM600. 2017. 92 p. Dissertação (Mestrado em Engenharia Florestal) - Universidade Federal do Paraná, Curitiba, 2017.

RIYAPHAN J, PHUMICHAI T, NEIMSUWAN T, WITAYAKRAN S, SUNGSING K, KAVEETA, $R$ et al. Variability in chemical and mechanical properties of Pará rubber (Hevea brasiliensis) trees. ScienceAsia, v. 41, p. 251-258, 2015. http://doi.org/2306/scienceasia1513-1874.2015.41.251

SILVA, J. C. Madeira preservada e seus conceitos. Revista da Madeira, ed. $\mathrm{n}=103$, março de 2007. Departamento de Engenharia Florestal. Universidade Federal de Viçosa- Viçosa - Minas Gerais. Disponível em: <http://www.remade.com.br/br/revistadamadeira_materia.php?num=1061\&subje $\mathrm{ct}=$ Preservantes\&title=Madeira $>$.

TRIANOSKI, R.; MATOS, J. L. M.; IWAKIRI, S.; PRATA, J. G. Avaliação da estabilidade dimensional de espécies de pinus. Floresta e Ambiente, v. 20, n. 3, p. 398-406, 2013. 